\title{
The Ethics of COVID-19 Risk Communication
}

\author{
Joseph H. Wu, PhD ${ }^{\top} \oplus$ and Stephen D. John, $P h D^{2}$ \\ ${ }^{1}$ The Warren Alpert Medical School, Brown University, Providence, RI, USA; ${ }^{2}$ Hatton Lecturer in the Philosophy of Public Health, Department of History \\ and Philosophy of Science, University of Cambridge, Cambridge, UK.
}

J Gen Intern Med 36(4):1092-3

DOI: $10.1007 / \mathrm{s} 11606-021-06600-3$

(c) Society of General Internal Medicine 2021

$\mathrm{W}$ ith the number of COVID-19 deaths continuing to rise, a central issue remains the mitigation of COVID-19 transmission risk. Policies mandating face masks, frequent testing, and social distancing constitute public health tools in achieving this end. However, another tool available that has received less attention is risk communication. The pandemic raises unique ethical challenges concerning how risk should be communicated to citizens.

Informing individuals of the risks of various behaviors is essential if citizens are to engage in containment strategies which cannot always be legally enforced, such as avoiding large gatherings without face masks. Limiting COVID-19 transmission depends on individuals acting prudently of their own volition. However, risk perceptions heavily influence decision-making and behaviors. Moreover, public risk perception is often misaligned with that of experts. During the 20092010 influenza pandemic, for example, public perception that the outbreak was exaggerated correlated with a reduced likelihood of adopting risk-reducing behavior change. ${ }^{1}$

Good risk communication is therefore critical to containing the pandemic. The World Health Organization (WHO) has issued guidance to support risk communication, which highlights the importance of proactive communication with the intended audience and the right of people to be informed of health risks. ${ }^{2}$ Likewise, the US Centers for Disease Control and Prevention (CDC) has recommended six core principles to guide "Crisis and Emergency Risk Communication": (1) be first, (2) be right, (3) be credible, (4) express empathy, (5) promote action, and (6) show respect. ${ }^{3}$ While these guidelines are instructive, risk communication raises deeper issues.

For example, discussions of risk communication typically assume two aims, which can come into conflict. ${ }^{4}$ One is to enable individuals to make informed decisions in line with their values. When communicating the risks and benefits of an

Received October 25, 2020

Accepted January 1, 2021

Published online January 25, 2021 operation, a surgeon's aim is to ensure the patient achieves the understanding necessary to make an informed choice about her treatment. The other is to encourage behavior change. In the context of cardiovascular disease, for instance, the aim of risk communication is often to mediate health-related behavior change. Consequently, what constitutes "effective" risk communication depends on whether communicators are seeking to fulfill a duty to inform or, instead, to persuade their audience of acting a certain way. These separate objectives of risk communication place emphasis on different ethical principles. Whereas the former emphasizes respect for autonomy, the latter stresses beneficence.

Determining how to balance these ethical values underlying the aims of risk communication becomes especially complicated in the COVID-19 context. In the clinic, a patient's decisions typically affect only that patient, as in the example of surgery. Insofar as individuals are most knowledgeable about what is best for them, risk communication should aim at promoting informed choice. But with COVID-19, any individual's decisions and resultant behaviors affect multiple people beyond herself. Risk communication must therefore consider the interests of these additional individuals and carefully assess how the values guiding communication should be adapted accordingly.

Some communicative strategies which would appear problematic in a clinical setting may become easier to justify in the context of COVID-19. For example, it is well documented that conveying relative risk reduction as opposed to absolute risk reduction leads to inflated risk perceptions. ${ }^{5}$ Communicating relative, rather than absolute, risk estimates to steer patients toward a particular treatment deemed good for them would be paternalistic in the clinic. Yet in the current pandemic, communicating relative risks to encourage behavior change essential to limiting COVID-19 transmission may be less problematic. One ethical justification for this is that such communication is not aimed at affecting an individual's self-regarding choices, but her other-regarding choices. By analogy, communicating the adverse effects of smoking with relative risks need not be paternalistic if the aim is to prevent the harms of secondhand smoke for others.

Another ethical complexity here concerns the very concept of risk. When assessing an individual's white blood cell count, there is one "true" white blood cell count being measured. But risk is different. There is no single "true" risk of COVID-19related mortality for each individual, because risk depends in 
part on how people are categorized. Since people can be categorized in many different ways, an individual's risk of COVID-19-related mortality will differ if she is categorized based on age alone, or age-and-location, or age-and-locationand-comorbidities, and so on. Unlike an estimate of white blood cell count, there is always more than one correct estimate of an individual's COVID-19-related mortality risk.

Hence, individual risk claims are always indexed to a description of that individual as a member of some subpopulation. But nature does not tell us how to categorize individuals. Rather, we make choices about how to classify individuals, which often reflect practical and ethical, rather than strictly scientific, concerns. The estimation of risk itself therefore partially depends on value judgments. To a certain extent, context can help guide the appropriate categorization. For the WHO, reporting risk based on age alone may be justifiable given the heterogeneity of the audience. But for a State Department of Health, reporting age-based risk alone would ignore ways of classifying data, such as particular counties disproportionately affected, crucial to guiding local decisionmaking. Understandably, the public might be confused when different agencies report apparently different risks. Communicating the most appropriate risk estimates while avoiding confusion or undermining public trust is an utmost priority.

Three implications of the preceding discussion are worth highlighting. First, risk communication is not merely a vehicle for transferring information, but also a tool to promote behavior change. A core ethical dilemma concerns whether, when, and to what extent the predictable effects of communicating a certain way can be leveraged to promote better outcomes.

Second, there is no perfectly neutral way to communicate risk. Unavoidable decisions such as the order of information or whether outcomes are framed positively or negatively influence the resulting perception of risk. Likewise, decisions about what risk estimate to communicate often reflect value judgments. For instance, communicating "the risk of dying from COVID-19" requires carefully conveying either (1) the risk of dying from COVID-19, if infected, the Infection Fatality Rate, or (2) the risk of dying from COVID-19, among people currently uninfected, the Population Fatality Rate. ${ }^{6}$ Both risk estimates may be true. Yet these subtle nuances in the definition of risk can have a large impact on public perceptions. Hence, deciding what risk to convey also raises tensions between the demands of beneficence and autonomy in risk communication.

Third, norms relevant to communicating risk in clinical contexts may not be a good guide to communicating risks of
COVID-19. Because individuals' responses to COVID-19 risk information impact others, respect for autonomy may take second place to ensuring individuals make the socially best decision. While deceptive risk communication simply to manipulate public behavior would be impermissible, there are many "shades" of encouraging behavior change and not all are ethically objectionable, all things considered. ${ }^{7}$

COVID-19 risk communication requires balancing duties to promote informed choice against the benefits of encouraging risk-reducing behavior change. Risk perceptions are vital to the adoption of recommended behaviors, and an accurate understanding of risk is central to enabling citizens to make informed choices beyond the scope of public health guidelines. Mitigating the COVID-19 pandemic hinges on good risk communication strategies. To inform the development of such strategies, evaluating the ethics of COVID-19 risk communication is an utmost priority.

Corresponding Author: Joseph $\mathrm{H}$. Wu, $\mathrm{PhD}$; The Warren Alpert Medical School, Brown University, Providence, RI 02903, USA (e-mail: joseph_wu@brown.edu).

\section{REFERENCES}

1. Rubin GJ, Amlôt R, Page L, et al. Public perceptions, anxiety, and behaviour change in relation to the swine flu outbreak: cross sectional telephone survey. BMJ. 2009;339:b2651.

2. World Health Organization. Risk communication and community engagement readiness and response to coronavirus disease (COVID-19): interim guidance. Available at: https://www.who.int/publications/i/item/riskcommunication-and-community-engagement-readiness-and-initial-response-for-novel-coronaviruses-(-ncov). Accessed October 5, 2020.

3. Centers for Disease Control and Prevention. Crisis and emergency risk communication overview for COVID-19. Available at: https://emergency. cdc.gov/cerc/training/webinar_20200406.asp. Accessed October 5, 2020.

4. Spiegelhalter D. Risk and uncertainty communication. Annu Rev Stat Appl. 2017;4:31-60.

5. Zipkin DA, Umscheid CA, Keating NL, et al. Evidence-based risk communication: a systematic review. Ann Intern Med. 2014;161:270-80.

6. Spiegelhalter D. What are the risks of COVID? And what is meant by "the risks of COVID'? Available at: https://medium.com/wintoncentre/whatare-the-risks-of-covid-and-what-is-meant-by-the-risks-of-covidc828695aea69. Accessed October 5, 2020.

7. Sunstein CR. The ethics of nudging. Yale $\mathrm{J}$ on Reg. 2015;32. Available at: https: / / digitalcommons.law.yale.edu/cgi/viewcontent.cgi? article $=1415 \&$ context=yjreg.

Publisher's Note: Springer Nature remains neutral with regard to jurisdictional claims in published maps and institutional affiliations. 